\title{
EFEITO DE ESCALA EXTRÍNSECO: COMO O AUMENTO DA ESCALA GEOMÉTRICA DE VIGAS DE CONCRETO AFETA SEU COMPORTAMENTO MECÂNICO
}

\author{
LEITE, FELIPE \\ Engenheiro Civil \\ Pontifícia Universidade Católica de Minas Gerais \\ Minas Gerais; Brasil \\ felipeccleite@gmail.com
}

\author{
SANTOS, AYRTON \\ Professor Adjunto \\ Pontifícia Universidade Católica de Minas Gerais \\ Minas Gerais; Brasil \\ ayrtonhugo@pucminas.br
}

\author{
ARAÚJO, WENDER \\ Engenheiro Mecânico \\ Pontifícia Universidade Católica de Minas Gerais \\ Minas Gerais; Brasil \\ wenderhsouza@gmail.com
}

\section{RESUMO}

O presente artigo apresenta uma abordagem crítica sobre a variação do comportamento mecânico de vigas de concreto obtidas por modelagem computacional, com auxílio do software Autodesk Robot ${ }^{\circ}$. A variação deste comportamento ocorreu pela ação simultânea de dois fatores que se relacionam entre si: o aumento das dimensões das vigas, por razão de semelhança, e a ação dos respectivos pesos próprios. $\mathrm{Na}$ análise realizada observou-se as variações no comportamento da flecha, do momento fletor e das tensões normal e cisalhante. Nesse ínterim, a influência que a microestrutura e os defeitos do material exercem sobre o comportamento mecânico não foram avaliados devido à própria metodologia do estudo, que norteou a obtenção de um modelo mínimo. Como resultado foi observada uma correlação matemática entre os fenômenos estudados e os fatores de escala utilizados. A partir dessa análise foi possível a concepção de um modelo matemático mínimo de representação dos fenômenos em questão.

Palavra-Chave: Efeito de escala, Comportamento mecânico, Tensão, Deformação.

\section{ABSTRACT}

This paper presents a critical approach to the mechanical behavior of concrete beams obtained through computational modeling using Autodesk Robot@ software. This variation of behavior was due to the action of two factors that are related to each other: the increase of the dimensions of the beams, by similarity, and the action of the respective weights. Variations in the behavior of deflection, bending moment and the normal and shear stress were analyzed. In the meantime, the influence that the microstructure and the defects of the material exert on the mechanical behavior were not considered due to the methodology of the study, which guided the obtaining of a minimum model. As result, a mathematical correlation was observed between the studied phenomena and the scale factors used. From this analysis it was possible to design a minimum mathematical model of representation of the phenomena in question.

Keywords: Size effect, Mechanical behavior, Stress, Deformation.

\section{INTRODUÇÃO}

\subsection{Definições}

Dois corpos constituídos pelo mesmo material, mas de tamanhos diferentes, possuem o comportamento mecânico diferentes entre si, conforme sugerido por BAZANT e PFEIFFER (1987).. Essa afirmação pode ser avaliada por meio da construção de modelos em escala, complementados por leis baseadas nas características dos materiais analisados.

Segundo KIM et al. (2007), o efeito de escala é representado pela combinação entre os aspectos microestruturais do material que compõe um determinado corpo e as características geométricas deste, discretizando-o em dois tipos: o efeito do tamanho do grão cristalino e o efeito do tamanho do espécime. Segundo REIS (2016) o efeito de escala pode ser separado em efeito de escala intrínseco e efeito de escala extrínseco. Nesta separação, o efeito de escala intrínseco 
está relacionado à influência da variação de tamanho da microestrutura sobre o comportamento mecânico da amostra, conservando-se a geometria deste. Já o efeito de escala extrínseco está associado à influência que a alteração das proporções do componente poderia exercer sobre seu comportamento mecânico.

\subsection{Histórico}

Apesar de pouco estudado, o efeito de escala extrínseco já foi do interesse de algumas personalidades seculares. CARNEIRO (1996) cita que Galileu Galilei (1564-1642), à sua época, mostrou grande interesse no comportamento mecânico de protótipos de modo a torna-los mais representativos e, a partir de seus estudos sobre a temática, chegou à conclusão de que quando se comparam dois corpos geometricamente semelhantes, as forças resistentes se relacionam com o quadrado da escala geométrica, ao passo que os pesos próprios se relacionam com o cubo dessa escala. Ainda, passando-se do modelo reduzido ao protótipo, a importância relativa do peso próprio cresce de tal maneira que ao ser atingido um tamanho limite a falha ocorre pela ação exclusiva de seu peso próprio, ao passo que o modelo de menor tamanho é capaz de suportar cargas adicionais, além do seu peso próprio. Além de Galileu Galilei, Isaac Newton (16431727) e Arquimedes de Siracusa (287a.c.-212a.c.) também se ocuparam com a análise de modelos reduzidos e posteriormente outros pesquisadores (BAZANT(1999) e KIM(2007)) aprofundaram estes estudos criando algumas hipóteses.

BAZANT (1999) explica que, em meados da década de 1970, o interesse da comunidade científica no efeito de escala em estruturas de concreto foi estimulado pela discrepância entre a metodologia laboratorial e o comportamento de grandes estruturas, como pontes e viadutos, na prática. Em tempos atuais, KIM et al. (2007) cita que o interesse da comunidade científica no assunto surgiu no início dos anos 1990, marcado pela atuação da indústria tecnológica na produção de componentes da microcomputação e robótica cada vez menores e mais potentes. Tal fato despertou a atenção de pesquisadores para os fenômenos que ocorriam quando reduzidas as escalas de corpos policristalinos, sendo constatado que, macroscopicamente, a medida em que se reduz a escala de um corpo também se reduz sua tensão de escoamento. Algumas hipóteses foram criadas para explicar e quantificar essa redução na tensão de escoamento, como a de MIYAZAKI et al. (1979) apud KIM et al. (2007), que cita a superfície do grão como uma região de concentração de tensões sendo que reduzir a escala da amostrafaz com que seja diminuída essa região de maior tensão. Semelhantemente, NAKAMASHI et al. (2000) apud KIM et al. (2007) explica que quando se aumenta a escala de um corpo também é aumentada a tensão atuante sobre este, sem o aumento da sua força resistente, que é derivada do atrito entre os cristais vizinhos, dando a entender que quanto maior a superfície específica dos cristais que compõe a amostra maior a força resistente do policristal.

\subsection{Objetivo}

O presente artigo analisa ao efeito de escala extrínseco, buscando quantificar de forma simples a resposta do comportamento mecânico de amostras de concreto não armado, obtidos por modelagem computacional, quando a escala varia em módulos pré-definidos, independente do arranjo cristalino. Trata-se da busca de um modelo matemático que generalize o fenômeno em questão e que expresse a física básica envolvida, também chamado de modelo mínimo. BATTERMAN (2002) reforça a utilização dessa abordagem devido à sua característica em fornecer soluções exatas e que, apesar de produzir um modelo altamente idealizado, ressalta que quanto mais detalhado um modelo mais ele tende a se distanciar da representatividade proposta, exigindo soluções por aproximação, enquanto que o modelo mínimo expressa a essência dos fenômenos modelados que se repete em padrões, não se atendo aos detalhes que diferenciam esses fenômenos e os tornam únicos.

\section{METODOLOGIA}

\subsection{Pré-processamento}

Este estudo é classificado como pesquisa numérica, na qual se relaciona a alteração de parâmetros geométricos e mecânicos aos efeitos resultantes desta alteração. No presente caso, o parâmetro principal é a geometria da seção transversal de cada viga, sendo esta alterada em determinadas escalas, juntamente com o comprimento. Deste modo, a escala de uma amostra de concreto é a variável independente enquanto o comportamento mecânico é a variável dependente. 
Foram utilizadas duas seções transversais, $20 \mathrm{~cm}$ x $20 \mathrm{~cm}$ x $100 \mathrm{~cm}$ (Figura 1) e $20 \mathrm{~cm}$ x $40 \mathrm{~cm}$ x $100 \mathrm{~cm}$ (Figura 2), e para cada uma destas foram determinadas cinco variações de escala, utilizando-se razão de semelhança e variando entre $1: 1 ; 2: 1 ; 3: 1 ; 4: 1 ; 8: 1$ :

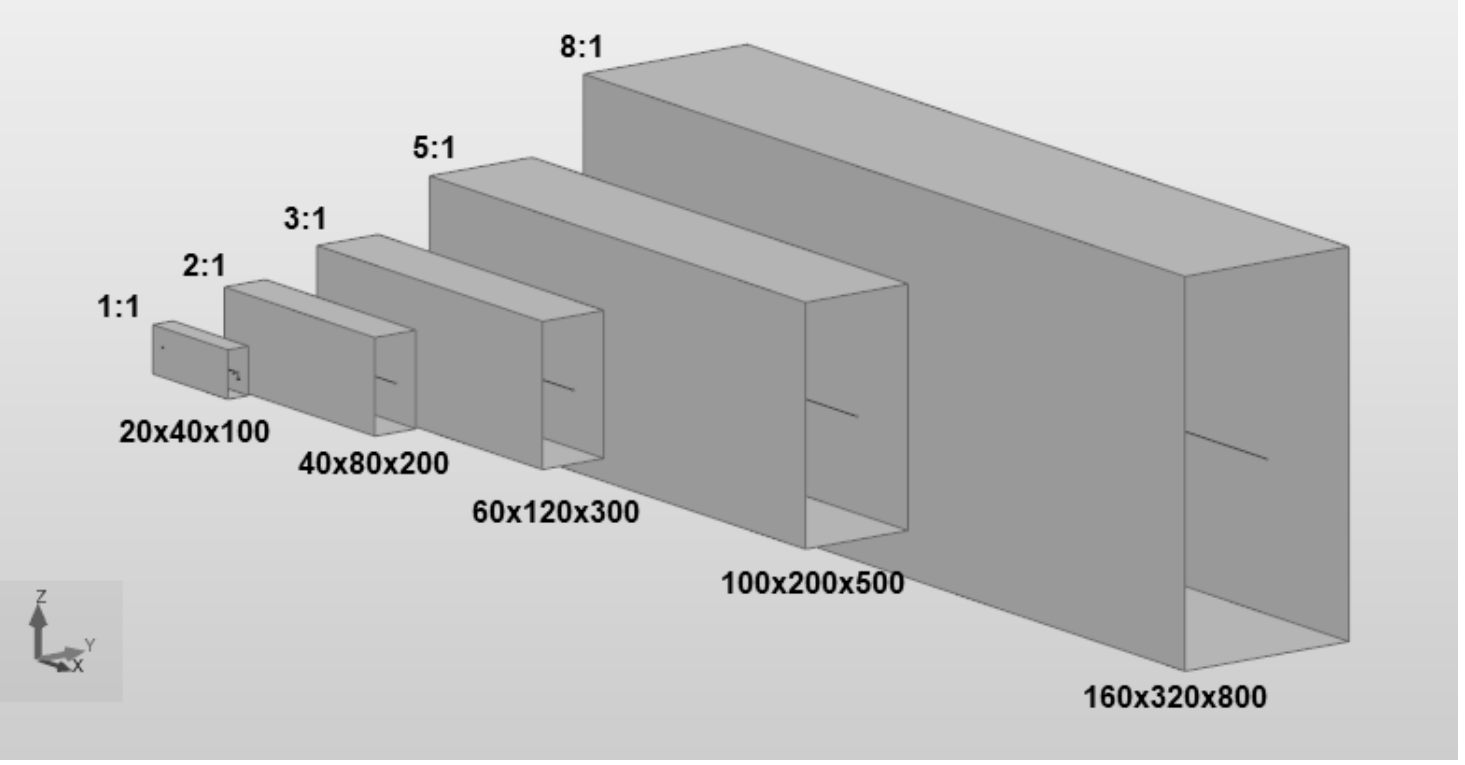

Figura 1: Vista em perspectiva do aumento da escala da viga $20 \mathrm{~cm}$ x $20 \mathrm{~cm}$ x $100 \mathrm{~cm}$, fora de escala.

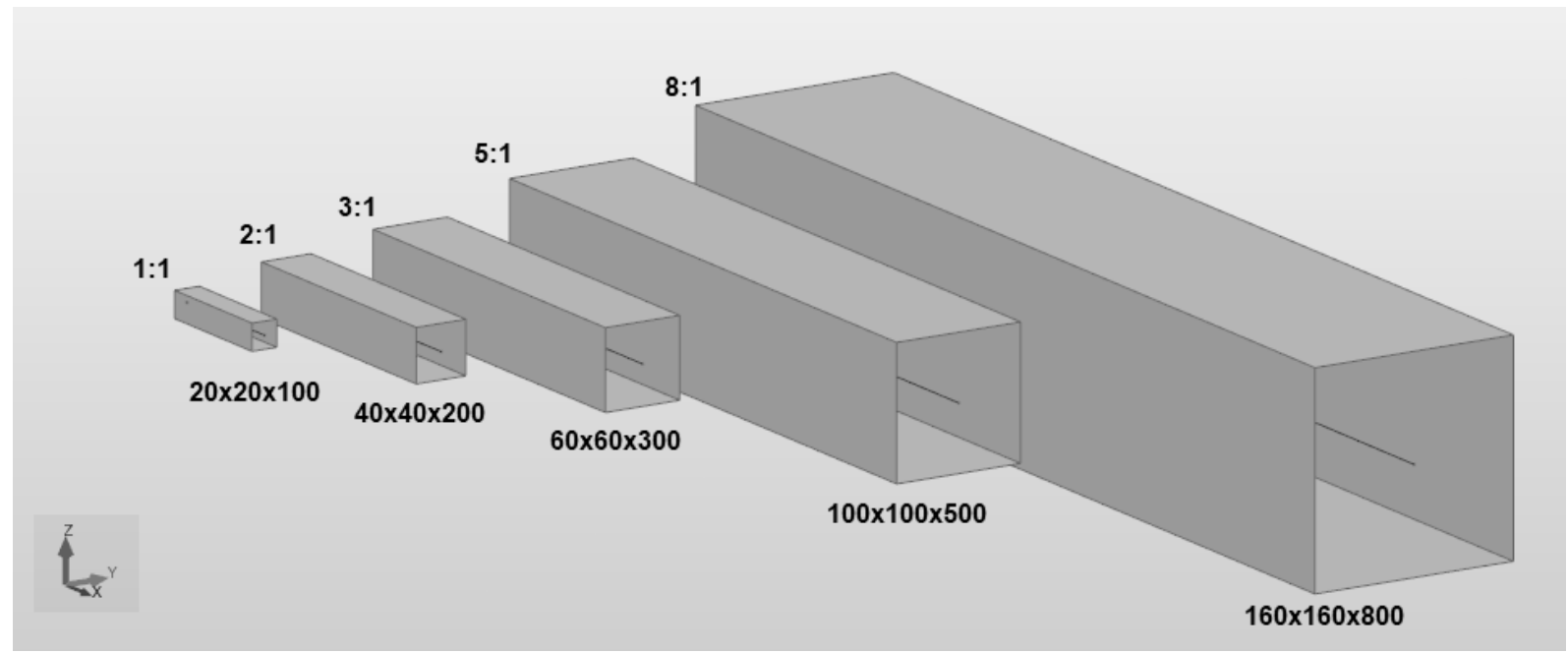

Figura 2: Vista em perspectiva do aumento da escala da viga $20 \mathrm{~cm} \mathrm{x} 40 \mathrm{~cm}$ x $100 \mathrm{~cm}$, fora de escala.

As propriedades do concreto utilizadas na modelagem são aquelas contidas na NBR 8953(2009). Ainda, considerou-se o concreto no regime elastoplástico e homogêneo, ou seja, não foram levados em consideração possíveis defeitos. Tal desconsideração adveio da própria metodologia deste estudo cuja buscou a concepção de um modelo numérico mínimo e que já foi citada.

- $\quad$ Massa específica seca: $2800 \mathrm{~kg} / \mathrm{m}^{3}$

- $\quad$ Classe de resistência: concreto C25

\subsection{Processamento}

Para a modelagem numérica foram utilizados os seguintes parâmetros, exigidos pelo software Autodesk Robot®:

I. Método de solução: linha do horizonte

II. Método iterativo

a. Número de incrementos de carga: 5

b. Número máximo de iterações para um incremento: 40 
c. Número de reduções de comprimento de um incremento: 3

d. Coeficiente de redução do incremento do comprimento: 0,500

III. Tipo de análise: Estática não linear

IV. Processo não linear convergente

a. Número total de incrementos de carga: 5

b. Número total de iterações: 10

\section{RESULTADOS E DISCUSSÃO}

Os resultados obtidos por meio do software Autodesk Robot® estão contidos na tabela 1:

Tabela 1 - Resultado da variação de escala das vigas, obtido por modelagem computacional

\begin{tabular}{|c|c|c|c|c|c|c|}
\hline \multirow{2}{*}{\multicolumn{2}{|c|}{ Perfil }} & \multicolumn{5}{|c|}{ Resultados } \\
\hline & & \multirow{2}{*}{$\begin{array}{c}\begin{array}{c}\text { Momento de } \\
\text { Inércia }\end{array} \\
{\left[\mathrm{cm}^{4}\right]}\end{array}$} & \multirow{2}{*}{$\begin{array}{c}\begin{array}{c}\text { Deslocamento } \\
\text { Máximo }\end{array} \\
{[\mathrm{mm}]}\end{array}$} & \multirow{2}{*}{$\begin{array}{c}\begin{array}{c}\text { Momento } \\
\text { fletor }\end{array} \\
\text { [kgf.m] } \\
\end{array}$} & \multirow{2}{*}{$\begin{array}{c}\begin{array}{c}\text { Tensão } \\
\text { Normal }\end{array} \\
{[\mathrm{MPa}]}\end{array}$} & \multirow{2}{*}{$\begin{array}{c}\begin{array}{c}\text { Tensão } \\
\text { Cisalhamento }\end{array} \\
{[\mathrm{MPa}]}\end{array}$} \\
\hline Tipo & Escala & & & & & \\
\hline \multirow{5}{*}{$20 \times 20$} & $1: 1$ & 13333,33 & 0,00342 & 12,51 & 0,092 & 0,018 \\
\hline & $2: 1$ & 213333,33 & 0,01369 & 200,11 & 0,184 & 0,037 \\
\hline & $3: 1$ & 1080000,00 & 0,03080 & 1013,05 & 0,276 & 0,055 \\
\hline & $5: 1$ & 8333333,33 & 0,08555 & 7816,76 & 0,460 & 0,092 \\
\hline & $8: 1$ & 54613333,33 & 0,21902 & 51227,93 & 0,736 & 0,147 \\
\hline \multirow{5}{*}{$20 \times 40$} & $1: 1$ & 106666,67 & 0,00086 & 25,01 & 0,046 & 0,018 \\
\hline & $2: 1$ & 1706666,67 & 0,00342 & 400,22 & 0,092 & 0,037 \\
\hline & $3: 1$ & 8640000,00 & 0,00770 & 2026,11 & 0,138 & 0,055 \\
\hline & $5: 1$ & 66666666,67 & 0,02139 & 15633,52 & 0,230 & 0,092 \\
\hline & $8: 1$ & 436906666,67 & 0,05475 & 102455,86 & 0,368 & 0,147 \\
\hline
\end{tabular}

Por análise, foi percebido um aumento relativo igual em cada característica mecânica com o aumento da escala, para cada uma das seções comerciais consideradas. Conforme observado na Tabela 1, houve um incremento igual a $100 \%$ na tensão normal das duas seções consideradas quando a escala passa de 1:1 para 2:1, assim como um incremento de $156 \%$ na deformação vertical quando a escala foi alterada de 5:1 para 8:1. Ou seja, constatou-se que as duas seções em questão, quando submetidas às mesmas condições, apresentam variações padronizadas e que se relacionam ao fator de escala utilizado.

Com isso, a partir deste padrão de variação, é possível se estabelecer uma relação matemática aplicável à uma seção qualquer, respeitando-se as condições apresentadas neste trabalho, com o intuito de se prever o comportamento mecânico de uma viga quando aumentada ou diminuída sua escala. Necessário esclarecer que os valores iniciais das características em questão devem ser obtidos de anteriormente ao processo.

Utilizando o fator de escala, ou razão de semelhança, como a variável independente e, chegou-se às seguintes relações matemáticas, apresentadas nas equações 1 a 5 :

$$
\begin{aligned}
f_{z, e} & =e^{2} \times f_{z, 0} \\
M_{y, e} & =e^{4} \times M_{y, 0} \\
I_{y, e} & =e^{4} \times I_{y, 0} \\
\sigma_{y, e} & =e \times \sigma_{y, 0} \\
\tau_{y, e} & =e \times \tau_{y, 0}
\end{aligned}
$$


$f_{z, e}$ : valor da deformação vertical após aumento ou diminuição da escala;

$f_{z, 0}:$ valor da deformação vertical inicial;

$e$ : fator de escala ou razão de semelhança;

$M_{y, e}$ : valor do momento fletor após aumento ou diminuição da escala;

$M_{y, 0}:$ valor do momento fletor inicial;

$I_{y, e}$ : valor do momento de inércia após aumento ou diminuição da escala;

$I_{y, 0}:$ valor do momento de inércia inicial;

$\sigma_{y, e}:$ valor da tensão normal após aumento ou diminuição da escala;

$\sigma_{y, 0}:$ valor da tensão normal inicial;

$\tau_{y, e}:$ valor da tensão de cisalhamento após aumento ou diminuição da escala;

$\tau_{y, 0}:$ valor da tensão de cisalhamento inicial.

Em relação à representatividade, o modelo obtido e exposto cumpre com seu objetivo de fornecer a matemática de base da resposta à variação da escala das vigas em questão. Se as diretrizes deste estudo incluíssem a heterogeneidade do concreto, com as imperfeições cristalinas e os macrodefeitos que, eventualmente, surgem durante o processo de hidratação do concreto, o modelo obtido provavelmente não forneceria dados precisos.

Segundo CALLISTER E RETHWISCH (2016), existência destas condições físicas atua, principalmente, diminuindo a resistência limite das peças. Além disso, SANTOS (2015) reforça que a heterogeneidade de um material é apontada como a principal causa da propagação lenta de fissuras estáveis que antecedem a ruptura. De fato, muitas das propriedades dos materiais são relativamente sensíveis a descontinuidades no perfil do material.

Neste estudo, as vigas modeladas apresentaram comportamento mecânico dentro do intervalo elástico devido à consideração da homogeneidade do material. Com isso, os valores que foram obtidos na modelagem numérica podem ser considerados como resultado da condição perfeita das vigas, ou seja, são produtos da utilização de um modelo mínimo. Infere-se, portanto, que a representatividade de qualquer modelo mínimo é condicional, restrita a fins educacionais e de pesquisa, e não como método de ensaio qualificador.

\section{CONCLUSÃO}

O presente estudo apresentou a modelagem computacional de vigas de concreto não armado submetidas ao peso próprio e uma análise sintética do comportamento destas quando aumentada a escala. Foram observadas variações padronizadas do comportamento mecânico nas três seções adotadas. Tal fato deve-se, principalmente, à consideração do concreto como material homogêneo, ou seja, foram desconsiderados os defeitos, tanto microestruturais quanto macroestruturais. Como resposta, foi obtido um modelo mínimo para análise do comportamento mecânico, de acordo com as premissas deste estudo.

Conclui-se, portanto, que o efeito de escala extrínseco, ou variação do comportamento mecânico de um corpo com o aumento de sua escala, poderia ser previsto por formulação matemática. Entretanto, para casos reais, nos quais se fazem presentes as descontinuidades inerentes ao processo de hidratação do concreto, os resultados podem se apresentar diferentes, sendo necessária a associação entre os pressupostos deste estudo com uma análise estatística. Apesar disso, para um corpo nas condições perfeitas, as relações matemáticas adquiridas são suficientes para a previsão da variação de comportamento mecânico.

\section{REFERÊNCIAS}

ASSOCIAÇÃO BRASILEIRA DE NORMAS TÉCNICAS. NBR 8953 2015: Concreto para fins estruturais Classificação pela massa específica, por grupos de resistência e consistência. Rio de Janeiro, 2008.

BATTERMAN, R. W. Asymptotics and the role of minimal models. The British Journal for the Philosophy of Science. Vol. 53, No.01, pp. 21-38. Mar. 2002. 
BAZANT, Z. P. Size effect on structural strength: a review. Archive of Applied Mechanics. No 69. Ed. Springer-Verlag, 1999.

Bazant, Z. e Pfeiffer, P. 'Determination of fracture energy from size effect and brittleness number'. ACI Materials Journal, vol. 84, pp. 463-480. 1987

CALliSTER, W. D.; RETHWISCH, D. G. Ciência e engenharia de materiais: Uma introdução. 9a ed. Editora LTC. 2016.

CARneIRO, F. L. Análise Dimensional e Teoria da Semelhança e dos Modelos Físicos. $2^{\mathrm{a}}$ ed. Ed. UFRJ. Rio de Janeiro, 1996.

KIM, G. Y.; NI, J.; KOÇ, M. Modeling of the Size Effects on the behavior of metals in microscale deformation processes. Journal of Manufacturing Science and Engineering. 2007. Disponível em: <www.researchgate.net/publication/236201709>. Acesso em: 10 dez. 2018.

MIYASAKI, S.; SHIBATA, K.; FUJITA, H.. Effect of specimen thickness on mechanical properties of polycrystalline aggregates with various grain sizes. Acta Metallurgica, Volume 27, Issue 5, May, 1979. Department of Materials Science and Engineering. Faculty of Engineering. Osaka University. Osaka, 1979.

NAKAMACHI, E.; HIRAIWA, K., MORIMOTO, H., HARIMOTO, M.. Elastic/Crystalline Viscoplastic Finite Element Analyses of Single- and Poly-Crystal Sheet Deformations and Their Experimental Verification. Osaka Insitute of Technology. International Journal of Plasticity, Volume 16, Issue 12. Osaka, 2000.

REIS, A. P. Efeito de escala na resistência mecânica de materiais. Dissertação. Instituto Superior Técnico. Lisboa, 2016.

SANTOS, A. H. A. Estudo experimental do efeito de escala em estruturas de concreto utilizando correlação de imagem digital. Tese de Doutorado. Programa de Pós-Graduação em Engenharia de Estruturas. Escola de Engenharia. Universidade Federal de Minas Gerais. Belo Horizonte, 2015. 